\title{
Lysmata seticaudata (Decapoda, Crustacea) als Putzergarnele im Mittelmeer
}

\author{
R. A. Patzner \\ Zoologisches Institut der Universität Salzburg; \\ Akademiestr. 26, A-5020 Salzburg, Osterreich
}

\begin{abstract}
Lysmata seticaudata (Decapoda, Crustacea) as cleaner shrimp in the Mediterranean Sea. Members of the shrimp species Lysmata seticaudata were observed to clean a morrey eel in the western Mediterranean Sea (Baleares, Spain). In contrast to the cleaning symbiosis of tropical shrimps, several Lysmata species have only one host, thus representing a primitive type of cleaning behaviour. Lysmata seticaudata is not dependent upon cleaning for food and usually these shrimps are found without a host.
\end{abstract}

\section{EINLEITUNG}

Von einer Putzsymbiose spricht man, wenn eine Tierart im Rahmen ihrer Nahrungsaufnahme eine andere von Ektoparasiten befreit. Die Putzsymbiose ist besonders für Fische wichtig, da es für sie sonst sehr schwer oder unmöglich ist, sich von ihren Hautparasiten zu befreien. In den meisten Fällen putzt ein spezieller Putzerfisch andere Fischarten (Matthes, 1978). Aus tropischen Meeresgebieten kennt man aber auch eine ganze Reihe von Putzergarnelen, die Fische säubern (Limbaugh et al., 1961; Feder, 1966; Holzberg, 1971; Mahnken, 1972; Bruce, 1976). Uber Putzergarnelen im Mittelmeerraum gibt es bisher nur zwei kurze Mitteilungen von Moosleitner (1979, 1980).

\section{METHODE}

Die Beobachtungen wurden tauchend mit einem Preßluftgerät durchgeführt. Photographiert wurde mit einer Nikonos IVA mit Vorsatzlinse (Hydro) und Elektronenblitz.

\section{ERGEBNISSE UND DISKUSSION}

An der Nordspitze von Ibiza (Balearen, Spanien) wurde in $15 \mathrm{~m}$ Tiefe eine Gruppe von etwa 10 Lysmata seticaudata beim Putzen einer Muräne (Muraena helena) beobachtet. Die Tiere saßen sowohl am Körper als auch am Kopf der Muräne, wo sie bis zur Mundöffnung vordrangen (Abb. 1). Vereinzelt wurden Garnelen auch im geöffneten Maul des Fisches beobachtet. Wurde die Muräne durch den Taucher gestört, zog sie sich in den hinteren Bereich der Höhle zurück. Je weiter sie sich zurückzog, desto mehr Garnelen ließen von ihr ab und plazierten sich in Guppen an der Höhlenwand. 


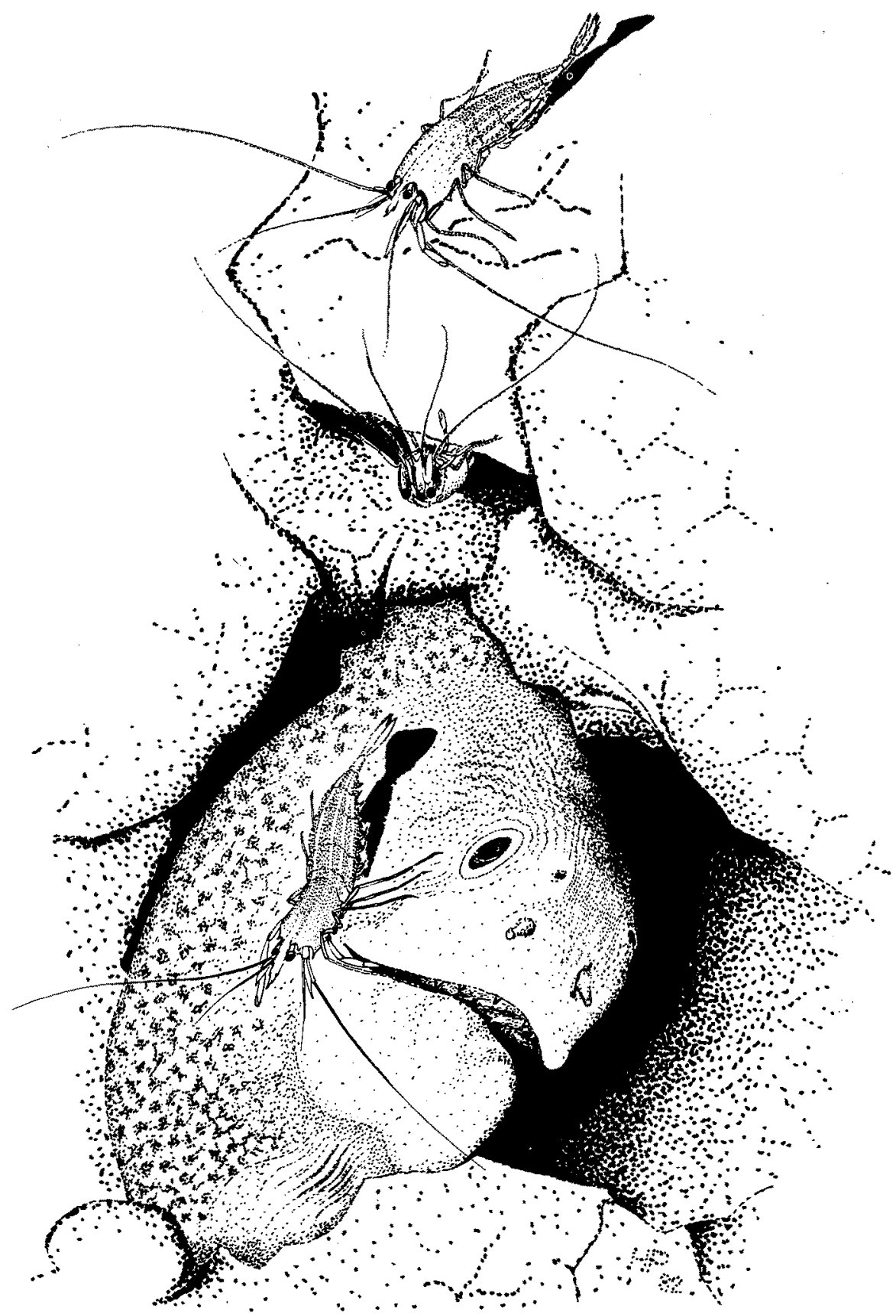

Abb. 1. Lysmata seticaudata beim Putzen am leicht geöffneten Maul einer Muräne (Muraena helena). Zwei weitere Lysmata an der Höhlenwand darüber. Zeichnung nach einer UnterwasserPhotographie 


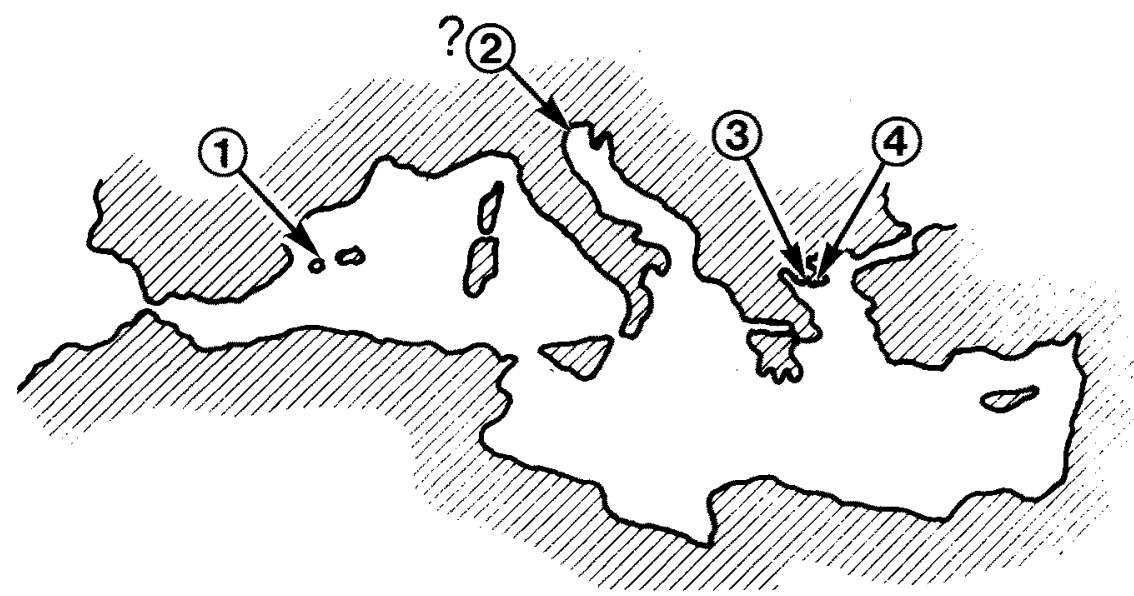

Abb. 2. Bisherige Beobachtungen von Lysmata seticaudata als Putzergarnele. $1=$ Nordspitze von Ibiza, Balearen; $2=$ Golf von Triest (Newton \& Stefanon, 1976); $3=$ Westküste von Longos, Chalkydike (Zieglmayer, pers. Mitteilung); $4=$ Westküste von Athos, Chalkydike (Moosleitner, 1979)

Die Muräne und die Lysmata-Garnelen waren während des Beobachtungszeitraumes von mehreren Wochen unter Tags immer in ihrer Höhle anzutreffen. Eine Beobachtung in der Nacht erfolgte nicht.

Fütterungsversuche ergaben, daß die Garnelen sich gierig auf angebotenes Futter (Loligo) stürzten. Das bedeutet, daß Lysmata nicht nur putzt, sondern auch andere Nahrung aufnimmt.

Im Laufe der Untersuchungen wurden vier weitere Muränen im selben Gebiet beobachtet. Bei zwei davon wurden keine Garnelen gesehen. Die Muränen zogen sich allerdings bei Annäherung des Tauchers so weit zurück, daß sie aus dem Blickfeld verschwanden. Eine Muräne bewohnte mit mehreren Lysmata ihre Höhle und eine andere mit einer weiteren Garnelenart, Stenopus spinosus.

Lysmata seticaudata wurde auch schon zusammen mit anderen höhlenbewohnenden Fischen im Mittelmeer gefunden. Eine Lysmata wurde im Golf von Triest mit einem Meeraal (Conger conger), allerdings nicht in Putztätigkeit, beobachtet (Newton \& Stefanon, 1976). In Griechenland (Marmaras, Chalkydike) wurden 5 Lysmata im seichten Wasser beim Putzen eines Schleimfisches (Blennius gattorugine) gesehen. Bei der Beobachtung, die um die Mittagszeit erfolgte, wurde die Kopfoberseite des Fisches geputzt (Zieglmayer, pers. Mitteilung). Abbildung 2 zeigt eine Zusammenstellung der bisherigen Beobachtungsstellen von Lysmata seticaudata als Putzergamelen im Mittelmeer.

Eine Reihe von tropischen Garnelen ist bereits als Fisch-Putzer beschrieben worden (Limbaugh et al., 1961; Holzberg, 1971). Sie haben gemeinsam, daß sie eine Putz"station" besitzen, zu der die Fische, angelockt durch die langen weißen Antennen der Garnelen, hinschwimmen, um sich von Ektoparasiten befreien zu lassen. Bisher.wurde nur eine einzige Species beschrieben, die von diesem Schema abweicht. Hippolysmata californica, die im gemäßigten Wasser von Kalifornien vorkommt, scheint ihre Fische 
nicht selbst anzulocken und repräsentiert damit einen sehr primitiven Typ des Putzverhaltens (Limbaugh et al., 1961). Ebenso ist das der Fall bei der im Mittelmeer beobachteten Lysmata seticaudata. Es ist anzunehmen, daß ein bestimmter Fisch (meist Muräne, aber auch andere) seine eigenen Putzergarnelen besitzt. Lysmata entfernt sich nur ungern von der schützenden Höhlenwand und ist deshalb nur bei solchen Fischen zu finden, die in engen Höhlen leben oder diese zumindest zeitweise aufsuchen.

Lysmata seticaudata ist im Mittelmeer relativ häufig ohne Putzkunden anzutreffen. Während des Tages verkriechen sich die Tiere in engen Schlupfwinkeln, treten jedoch in der Nacht in ganzen Gruppen in den Höhlenräumen auf (Riedl, 1966; CouturierBhaud, 1974). Abbildung 2 zeigt, daß Lysmata seticaudata sowohI im westlichen als auch im östlichen Mittelmeer und in der Adria (?) ihre Putztätigkeit ausübt. Aus dieser Tatsache kann man schließen, daß sie im ganzen Mittelmeergebiet putzt.

Um herauszufinden, wie sich diese Art von Putzsymbiose entwickelt hat, wäre es wichtig, die Frage zu klären, ob der Fisch sich eine Höhle, die bereits von Lysmata bewohnt ist, aussucht oder umgekehrt. Ein rein zufälliges Zusammentreffen ist bei der Seltenheit der Beobachtungen allerdings nicht auszuschließen.

Zwei Arten der Gattung Stenopus (St. hispidus und St. scutellatus) sind Putzergarnelen der tropischen Meere (Feder, 1966). In einer weiteren Untersuchung soll geklärt werden, ob die im Mittelmeer lebende Stenopus spinosus ebenfalls Fische putzt (Patzner, in Bearbeitung).

Danksagung. Ich danke der "Stiftungs- und Förderungsgesellschaft der Paris Lodron-Universität in Salzburg" für die Unterstützung und Prof. L. B. Holthuis (Leiden) für Hinweise.

\section{ZITIERTE LITERATUR}

Bruce, A. J., 1976. Shrimps and prawns of coral reefs, with special reference to commensalism. In: Biology and geology of coral reefs. Ed. by O. A. Jones \& R. Endean. Acad. Press, New York, 3, $37-94$.

Couturier-Bhaud, Y., 1974. Cycle biologique de Lysmata seticaudata Risso (Crustace, Decapode). I. Cycle biologique des animaux adultes. - Vie Milieu 3, 413-422.

Feder, H. M., 1966. Cleaning symbiosis in the marine environment. In: Symbiosis. Ed. by S. M. Henry. Acad. Press, New York, 1, 327-380.

Holzberg, S., 1971. Beobachtung einer Putzsymbiose zwischen der Garnele Leandrites cyrtorhynchus und Riffbarschen. - Helgoländer wiss. Meeresunters. 22, 362-365.

Limbaugh, C., Pederson, H. \& Chace, F. A., 1961. Shrimps that clean fishes, - Bull. mar. Sci. Gulf Caribb. 11, 237-257.

Mahnken, C., 1972. Observations on cleaner shrimps of the genus Periclimenes. - Bull. nat. Hist. Los Angeles County 14, 71-83.

Matthes, D., 1978. Tiersymbiosen und ähnliche Formen der Vergesellschaftung. - Fischer, Stuttgart, $241 \mathrm{pp}$.

Moosleitner, H., 1979. Putzergarnelen im Mittelmeer. - Aquarien Magazin 12, 603.

Moosleitner, H., 1980. Putzerfische und -garnelen im Mittelmeer. - Zool. Anz. 205, 219-240.

Newton, R. S. \& Stefanon, A., 1976. Primi resultati dell'uso simultaneo in Alto Adriatico di side-scan sonar, sub-battom profiler ed ecografo. - Mem. biogeogr. adriat. (Suppl.) 9, 33-66.

Riedl, R., 1966. Biologie der Meereshöhlen. - Parey, Hamburg, 636 pp. 